\title{
Geostatistical visualisation and spatial statistics for evaluation of the dispersion of epidemic highly pathogenic avian influenza subtype H5N1
}

\author{
Michael P. WARD ${ }^{1 *}$, Daniel MafteI ${ }^{2}$, Cristian Apostu ${ }^{3}$, Andrian Suru $^{4}$ \\ ${ }^{1}$ College of Veterinary Medicine \& Biomedical Sciences, Texas A\&M University, College Station, Texas, USA \\ ${ }^{2}$ Regional Diagnostic Laboratory (DSVSA), Tulcea, Romania \\ ${ }^{3}$ Institute for Diagnosis and Animal Health (IDSV), Bucharest, Romania \\ ${ }^{4}$ National Animal Health and Food Safety Authority (ANSVSA), Bucharest, Romania
}

(Received 1 September 2007; accepted 9 November 2007)

\begin{abstract}
The aim of this study was to evaluate a range of statistical and geostatistical methods for their usefulness in providing insights into how highly pathogenic avian influenza (HPAI) subtype H5N1 might spread through a national population of village poultry. The insights gained allow the generation of disease dispersion hypotheses. The case study data set consisted of 161 outbreaks of HPAI subtype H5N1 in village poultry reported in Romania between October 2005 and June 2006. Reports of village outbreaks (\%) occurred in three waves: October-December (14\%), February-March (16\%), and May-June (68\%). Risk mapping - based on variography and kriging - was used to visualize the evolution of the epidemic. Outbreaks first appeared in eastern and southern Romania, particularly within an area that forms part of the Danube River Delta. The largest phase of the epidemic affected villages in all parts of central, southern, and eastern Romania, but outbreaks were clustered in central Romania. Outbreaks spread in an east to west direction. By using geostatistical visualisation and spatial statistics, the evolution of the epidemic could be characterised into two parts: disease introduction, local spread, and sporadic outbreaks, and long-distance disease spread with rapid epidemic propagation. This is consistent with the hypothesis that the environment and landscape (specifically the Danube River Delta) played a critical role in the introduction and initial spread of HPAI subtype H5N1 during the autumn and winter of 2005, and that the movement of poultry might have introduced the infection into central Romania during the spring and summer of 2006. Further research focusing on the spatio-temporal interface between the two parts of the epidemic might reveal how and why it progressed from a confined, local epidemic to a large, national epidemic. Such information would assist efforts to limit the global spread of HPAI subtype H5N1.
\end{abstract}

avian influenza H5N1 / poultry / epidemic / geostatistical / Romania

\section{INTRODUCTION}

Highly pathogenic avian influenza (HPAI) virus subtype $\mathrm{H} 5 \mathrm{~N} 1$ is an emerging issue for world health: it has caused numerous disease outbreaks in domestic poultry and wild bird populations, and threatens human health. Between 1997 and 2007, a total of 312 cases of human infection has been reported to the World Health Organization, resulting in

* Corresponding author: mward@cvm.tamu.edu
$190(61 \%)$ deaths in 12 countries in SouthEast Asia, China, central Asia, the Middle East, and Africa ${ }^{1}$. There is a fear that H5N1 could become the next pandemic influenza strain [1]. Because exposure to sick or dead

\footnotetext{
${ }^{1}$ World Health Organization, Cumulative number of confirmed human cases of avian influenza A/(H5N1), reported to WHO, 12 June 2007, http:// www.who.int/csr/disease/avian_influenza/country/ cases_table_2007_06_12/en [consulted 16 June 2007].
} 
poultry is a strong risk factor for human disease caused by HPAI subtype H5N1 [9], the threat of pandemic flu can be reduced by more effectively controlling the spread of HPAI through national poultry flocks. The first step in controlling the spread of HPAI subtype $\mathrm{H} 5 \mathrm{~N} 1$ is to understand how it spreads once introduced to a country. Epidemic data provide a valuable insight into such disease spread patterns.

Avian influenza virus infection is endemic in a range of free-living bird species worldwide [1], particularly species associated with water - Anseriformes (ducks, geese, swans) and Charadriiformes (gulls, terns, shorebirds) [25]. Waterfowl can be infected by all subtypes of type A influenza viruses, with few or no clinical signs [33]. These species are capable of spreading influenza viruses between regions [13].

Research suggests that waterfowl and shorebirds maintain a separate reservoir of viral gene pools from which new virus subtypes emerge [32]. In the recent outbreaks of HPAI subtype H5N1 in waterfowl, the virus has been more prevalent in respiratory tissue than intestinal tissue [26]. Persistence of this strain in migratory waterfowl (or even the environment) could be due to the prolonged shedding of virus in ducks, as well as changes in the virus that allows transmission via respiratory routes [26]. Prior to the recent HPAI subtype H5N1 outbreaks in ducks, host adaptation and lack of virulence in aquatic birds was cited as the reason for persistence in these species [32]. Recent studies in China have shown that viral lineages, established in poultry, have infected ducks $[15,26]$.

In the northern hemisphere, avian influenza virus infection rates are highest during spring migration for gulls and shorebirds, whereas waterfowl infections peak in late summer and early fall [13]. Juvenile waterfowl are more susceptible to infection; when the birds are migrating south, a higher prevalence is observed than in the spring, when the juveniles have matured [8].

HPAI outbreaks in poultry are sometimes assumed to occur from contact with wild avian species $[12,17,27]$. The HPAI subtype H5N1 currently circulating in Asia may have arisen from a goose virus in Guangdong, which then infected poultry and reassorted with a H9N2 and/or a H6N1 subtype virus. When the HPAI virus subtype $\mathrm{H} 5 \mathrm{~N} 1$ infected humans in Hong Kong in 1997, several deaths were reported. The strain was eradicated from poultry, but it continued to circulate in geese. In 2002, the haemagglutinin (HA) gene showed antigenic drift - especially in the $\mathrm{Z}$ genotypes, found in chickens, humans, and aquatic poultry [31]. This genotype is pathogenic in ducks, causing neurological disease and death; in other cases, no clinical disease was observed. Traditional husbandry practices in China and Southeast Asia, which favour commingling of multiple avian and mammalian species - as well as the popularity of live bird markets - may have contributed to the maintenance of the H5N1 virus strain [24]. In other regions of the world, HPAI outbreaks in commercial poultry have been traced to live bird markets $[6,10,16]$.

Outbreaks of transboundary diseases, such as HPAI, provide a unique insight into how animal diseases spread through space and time. Through the information generated, a better understanding of the underlying causes of the disease process can be gained, facilitating the design of disease prevention programs and surveillance systems.

The aim of this study was to evaluate a range of spatial statistical and geostatistical methods for their usefulness in providing insights and generating hypotheses regarding how HPAI subtype H5N1 might spread through a national population of village poultry. To achieve this aim, an epidemic disease data set describing 161 village poultry outbreaks of HPAI subtype H5N1 in Romania between 2005 and 2006 was used. Several techniques that have not widely been applied to analyse such data, including directional tests, local cluster tests, and kriging, were evaluated. The primary null hypothesis tested was that outbreaks of HPAI subtype H5N1 in Romania between 2005 and 2006 were randomly distributed in time and space. The secondary null hypothesis tested was that the spatial distribution of outbreaks during

Page 2 of 12 (page number not for citation purpose) 
temporal phases of the epidemic were the same.

\section{MATERIALS AND METHODS}

The first outbreak of HPAI subtype H5N1 was detected in Romania in early October 2005. The last outbreak was reported in early June 2006. Data available for confirmed outbreaks included the outbreak location ( $x, y$ coordinates), county and date of occurrence. An epidemic curve was constructed by counting the number of outbreaks reported per week between October 2005 and June 2006. The median epidemic date and epidemic interquartile range (dates between which $50 \%$ of outbreaks were reported) were calculated (Statistix 8.0, Analytical Software, Tallahassee, FL, USA).

Reported outbreaks were mapped using Dealul Piscului 1970 datum and a Stereographic 70 projection system (ArcGIS ${ }^{\mathrm{TM}}$ 9.0, ESRI Inc., Redland, CA, USA). The mean center and deviational ellipse of these outbreaks was calculated (ArcGIS $^{\text {TM }}$ 9.0 Spatial Statistics, ESRI Inc.); these descriptive statistics were also estimated, weighted by the number of days since the first outbreak was reported (7 October 2005). In addition, descriptive statistics were also estimated for each of the epidemic phases identified.

A directional statistic [11] was used to determine if there was a systematic, directional spread of outbreaks through Romania during the epidemic period. A chain of infection was constructed by first sequencing the outbreaks by date of occurrence (the primary outbreak first, followed by the second outbreak, and so on). A line was then drawn to connect the location of the first outbreak to the location of the second outbreak, repeating this until all outbreaks were connected. This chain of infection had two ends (the first and last outbreaks) and branches when outbreaks occurred on the same date. Various possible "chains of infection" were investigated by specifying the time connection matrix as relative (each outbreak connected to all of the outbreaks that followed it), adjacent (each outbreak connected only to its temporal nearest neighbouring outbreak) and following (each outbreak connected only to the outbreaks that immediately followed it). The test statistic is a vector whose direction is the average direction of the links making up the chain of infection, and whose magnitude is the angular variance of these links. When these links all point in the same direction, the angular variance is small; when they point in many directions the angular variance is large. The significance of the test statistic was estimated by Monte Carlo simulation, using ClusterSeer version 2.0 (TerraSeer Inc. 2002, Ann Arbor, MI, USA).

The spatio-temporal distribution of outbreaks was described by Moran's autocorrelation statistic (ArcGISTM 9.0 Spatial Statistics), in which the outcome of interest was outbreak day. Variography (Variowin 2.0. University of Lausanne, Switzerland) and kriging (ArcGISTM 9.0 Spatial Analyst) was also used to visualize the spatio-temporal distribution of the epidemic. Outbreaks reoccurred at four locations. All possible unique pairs of outbreak locations ( $n=12880)$ were formed using all unique outbreak locations $(n=161)$. A matrix of Euclidean distances and date (day) of outbreak was calculated and a semivariogram was formed. A semivariogram is a plot of the semivariance of all pairs of locations at a series of defined distances (lags) [28]. For locations close to each other, values (for example, the epidemic day of reporting in this study) are expected to be similar and the semivariance will be low. For example, within a local neighborhood, if a disease is contagious outbreaks are expected to be reported from these locations within a relatively narrow window of time. Thus, epidemic day values will be highly correlated. As locations get farther apart, values are expected to become more dissimilar (for example, reported outbreak day will vary greatly) and thus the semivariance increases. The rate of increase in semivariance as distance increases (nugget and sill), and the distance at which locations can essentially be considered independent (range), characterizes the spatial pattern of the event-of-interest. A range of lag numbers and lag spacings were chosen to produce a semivariogram which could be described by one of a number of a priori models. Several statistical models (exponential, Gaussian, spherical, power) were investigated to identify a line of best fit. Using the line-of-best-fit approach, the parameters of the selected model (nugget, range, sill) were estimated. Estimating the parameters of the line of best fit of an empirical semivariogram allows the distribution to be modeled and interpolated with techniques such as kriging. The estimated parameters were used to produce an interpolated map of outbreak day. These maps were overlaid on the location of outbreaks to identify localities (rather than individual villages) where outbreaks were reported early 
during the epidemic. Identification of areas where epidemic disease first occurs can provide clues to the mechanisms of disease introduction and subsequent spread. Such information can be applied to the design of disease prevention programs, disease surveillance and risk assessments.

Local clusters of outbreaks were investigated by estimating Anselin's local indicator of spatial autocorrelation statistic (ArcGISTM 9.0 Spatial Statistics). Spatial weights were defined by the inverse of Euclidean distance squared, and were globally standardized. Reported outbreaks were also examined (SaTScan version 6.1.2, www.satscan.org) for clusters in time and space using the scan statistic space-time permutation model [14]. Outbreak data - location $[x, y]$ and reported date of outbreaks $[z]$ - were analysed. In the space-time permutation model, the number of observed cases in a cluster is compared to what would have been expected if the spatial and temporal locations of all cases were independent of each other, that is, no space-time interaction [14]. The data were only scanned for clusters with excess number of outbreaks: areas in Romania that, during a specified time period, had a high proportion of village outbreaks compared to surrounding areas. We assumed that the number of villages in Romania (the population at-risk) remained stable during the nine-month study period. A scanning window of 14 days and $10 \mathrm{~km}$ radius was used to reflect potential disease spread. Monte Carlo replications were used to estimate the statistical significance of the likelihood ratio statistics of the clusters identified (SaTScan version 6.1.2).

\section{RESULTS}

During the epidemic period (243 days), a total of 165 outbreaks were detected in 25 of the 41 counties (judete) of Romania. The mean number of outbreaks per county was four. The largest number of outbreaks were reported from Brasov (29) and Prahova (28) in central Romania, Bacau (14) in northeast Romania, and Constanta (14) and Tulcea (11) in eastern Romania. The median epidemic date was 18 May (day 224 of the epidemic). Half of all outbreaks were reported during an 85day period, between 27 February and 23 May (epidemic days 144-229). Visual inspection of the epidemic curve (Fig. 1) revealed three

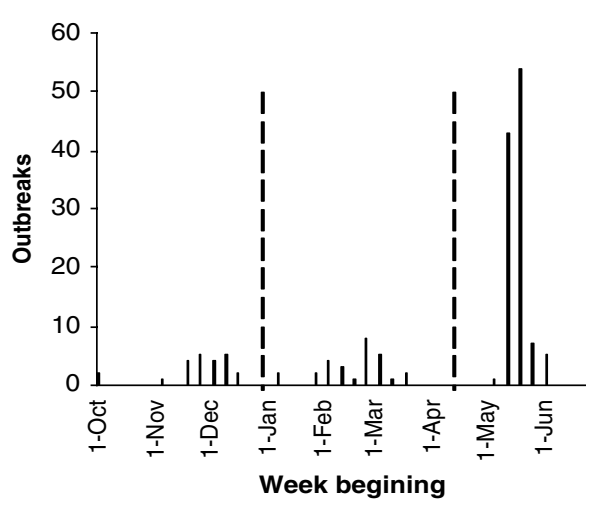

Figure 1. Epidemic curve of highly-pathogenic avian influenza subtype H5N1 outbreaks in Romania, October 2005 - June 2006. The three phases of the epidemic are indicated by dashed lines.

epidemic phases: 7 October-29 December 2005 (days 1-84), 30 December 200516 April 2006 (days 85-192), and 17 April6 June 2006 (days 193-243).

Since outbreaks reoccurred at four locations, spatial analysis was restricted to a total number of 161 unique outbreak locations. The mean distance between outbreak locations was $9.5 \mathrm{~km}$. The mean center $(x=603446, y=$ 416655) of reported outbreaks in Romania was $29 \mathrm{~km}$ north-east of the mean center ( $x=577067, \quad y=428360)$ of outbreaks weighted by outbreak day. The mean centers of outbreaks reported in the first and second epidemic phases $(x=721033, y=376624$ and $x=715981, y=333656$, respectively) were located $43 \mathrm{~km}$ apart in eastern Romania. The mean center of outbreaks reported in the third epidemic phase $(x=551993, y=$ 444363) was located in central Romania, $58 \mathrm{~km}$ north-west of the mean center of all reported outbreaks. Standard deviational ellipses calculated for these three epidemic phases increased in temporal sequence (7 713, 10370 , and $13810 \mathrm{~km}^{2}$, respectively), but their shape (latitude:longitude standard deviation ratio $2.12,2.80$, and 1.24 , respectively) and angle $\left(283,275\right.$, and $\left.319^{\circ}\right)$ varied (Fig. 2): the first two epidemic phases were spatially-distributed east-west, whereas the 


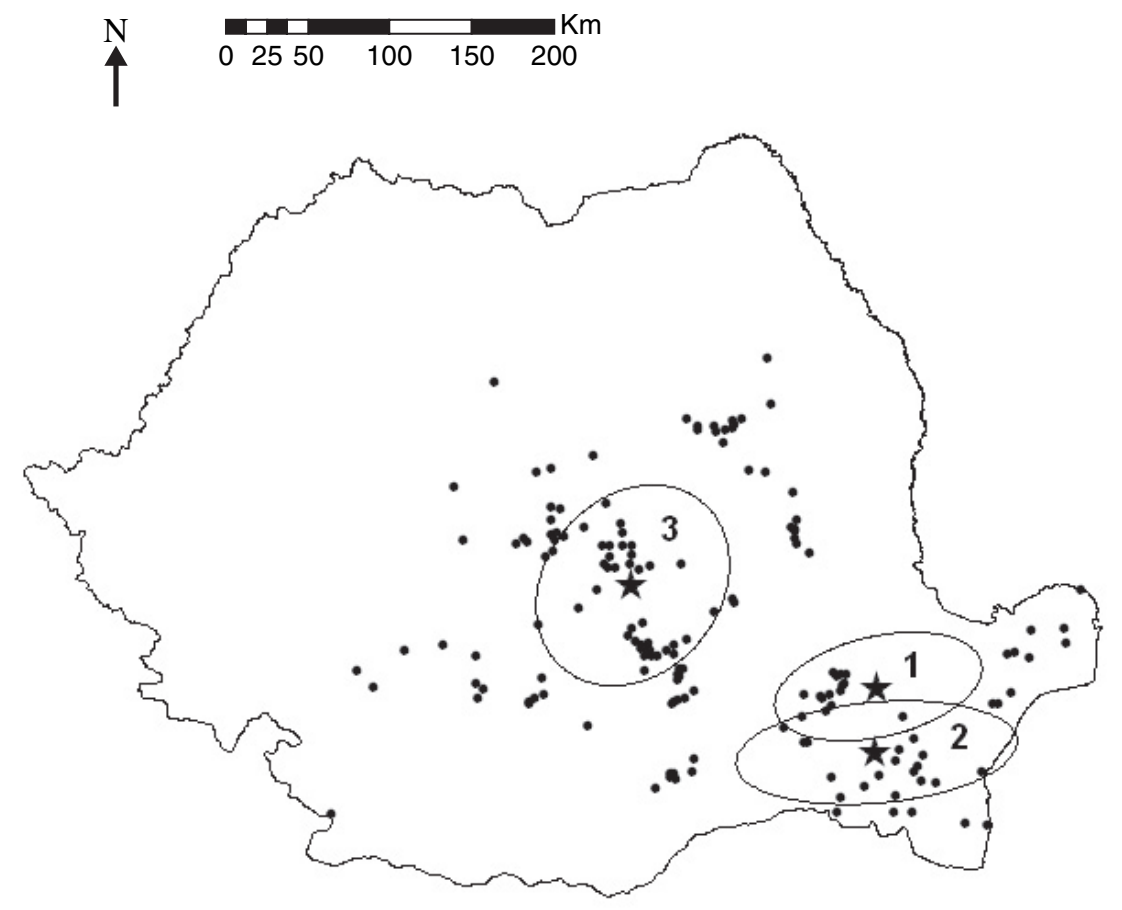

Figure 2. Distribution of highly-pathogenic avian influenza subtype H5N1 outbreaks $(\bullet)$ in Romania, October 2005-June 2006. The mean centers $(\star)$ and standard deviational ellipses of the three epidemic phases (1: 7 October-29 December 2005; 2: 30 December 2005-16 April 2006; 3: 17 April-6 June 2006) are shown.

third phase displayed a more regular circular distribution.

A significant $(P<0.001)$ epidemic direction was detected only when the outbreak time connection matrix was assumed to be relative (each outbreak connected to all of the outbreaks that followed it). For all outbreaks, the average angle of spread was $282^{\circ}$ (east to west), although the angular concentration (0.314) indicated moderate variation in the direction of spread. Outbreaks reported during phases 1 and 2 of the epidemic and phase 3 showed very similar $(0.239$ and 0.237 , respectively), significant $(P<0.001)$ angular concentrations, and the angular direction of each of these sub-epidemics was southerly (167$\left.177^{\circ}\right)$, in contrast to the westerly $\left(282^{\circ}\right)$ angular direction of the overall epidemic.

The semivariogram of outbreak day, using 15 lags and a lag size of $25 \mathrm{~km}$ (Fig. 3) was best fit by a spherical model, with an estimated nugget, range, and partial sill of $3.6,190 \mathrm{~km}$ and 4.32, respectively. This model implies that the reported epidemic day of outbreaks located within a distance of approximately $190 \mathrm{~km}$ are correlated, and that approximately $55 \%$ of the variance in reported epidemic day might be explained by the location of outbreak. The resulting map of interpolated outbreak day (Fig. 4) clearly shows the occurrence of H5N1 outbreaks in eastern Romania during the first 120 days of the epidemic.

The distribution of outbreaks was strongly clustered $(I=0.74, P<0.001)$ by outbreak day. A significant cluster of outbreaks in southeastern Romania was identified by the local indicator of spatial autocorrelation (LISA) statistic (Fig. 5). In this area, outbreaks occurred in 11 villages within a distance of approximately $65 \mathrm{~km}$ between 20 November 


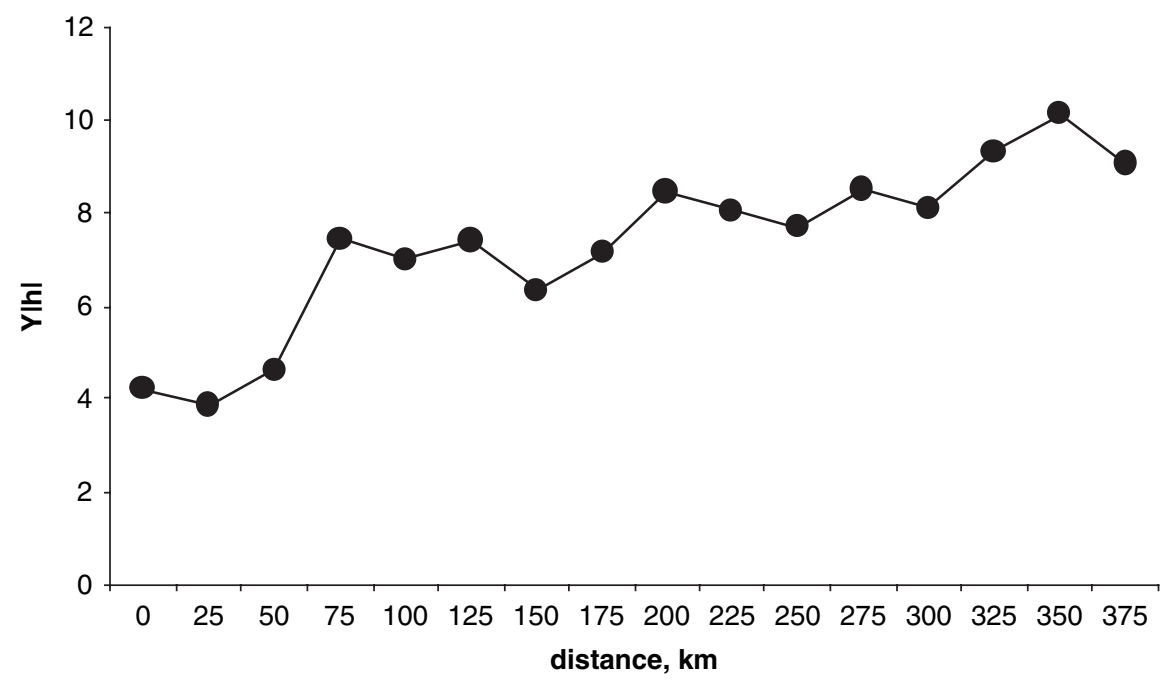

Figure 3. Semivariogram of the day of highly-pathogenic avian influenza subtype H5N1 village outbreak in Romania, October 2005-June 2006.

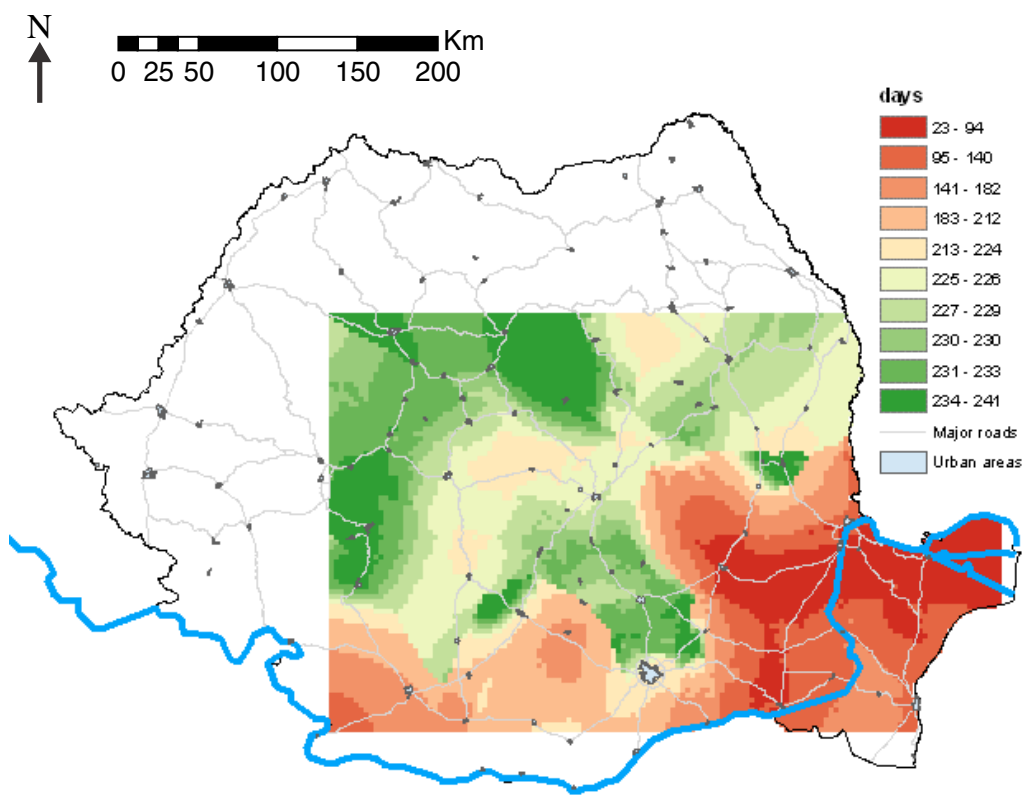

Figure 4. Interpolated day of highly-pathogenic avian influenza subtype H5N1 village outbreak in Romania, October 2005-June 2006. The Danube River is shown.

Page 6 of 12 (page number not for citation purpose) 


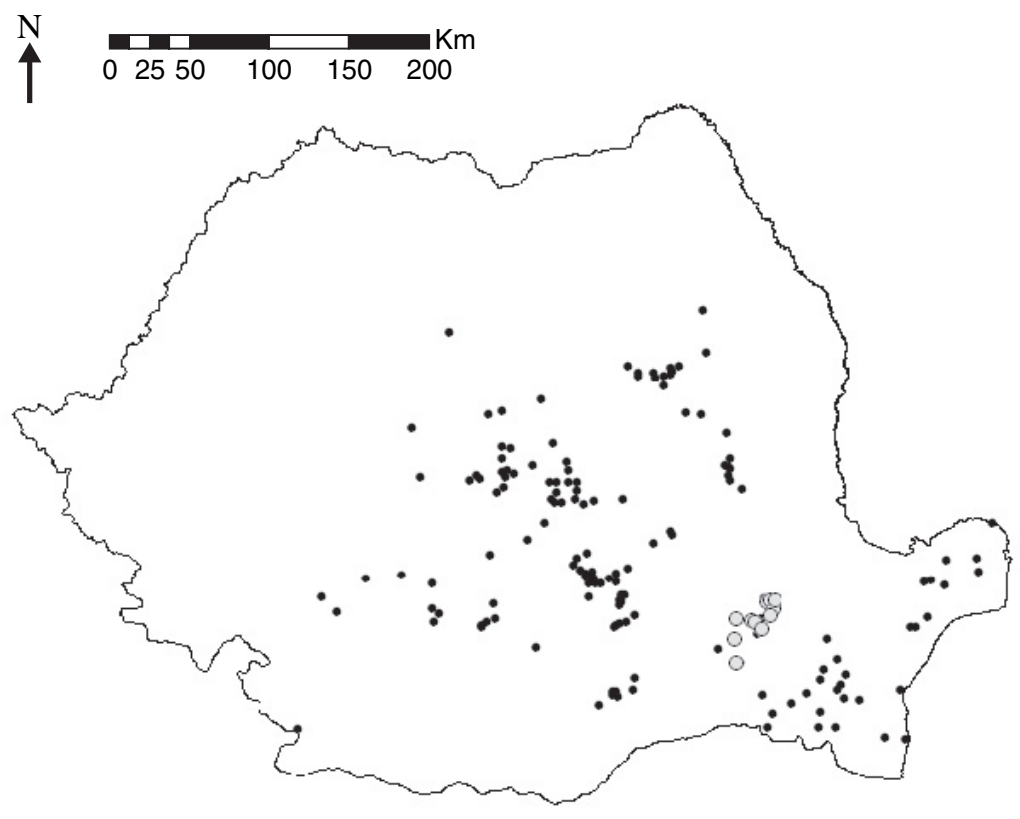

Figure 5. A cluster ( $(0)$ of highly-pathogenic avian influenza subtype H5N1 village outbreaks $(\bullet)$ in Romania, October 2005-June 2006, identified by the local indicator of spatial autocorrelation statistic.

and 15 December 2006 (epidemic days 45 and 70).

Three significant $(P<0.05)$ spacetime clusters of outbreaks were identified (Fig. 6). The most likely cluster $(P=0.029)$ included ten villages located in Constanta county, south-east Romania ( $x=751967$, $y=319610$; radius $8733 \mathrm{~m})$. Within this cluster, three outbreaks were reported between 27 February and 2 March 2006. Based on the area of the cluster and the time period, only 0.07 outbreaks would be expected to be reported. Other, significant $(P=0.03)$ clusters identified were located in Brasov county, central Romania (11 villages: $x=492542$, $y=485538$, radius $8,597 \mathrm{~m}$; four outbreaks reported between 2 and 15 May 2006, 0.22 outbreaks expected) and Braila county, southeast Romania (six villages: $x=695960$, $y=387007$, radius $7263 \mathrm{~m}$; four outbreaks reported between 18 November and 1 December 2005, 0.22 outbreaks expected).

\section{DISCUSSION}

Outbreaks of HPAI subtype H5N1 in Romania between 2005 and 2006 were not randomly distributed in time and space. Furthermore, differences in the spatial distribution of outbreaks during temporal phases of the epidemic were identified, which might suggest differences in the mechanism of disease spread.

Highly pathogenic avian influenza virus can be introduced into populations of domestic poultry from wild waterfowl. Spread of virus within populations of domestic poultry is likely to be via the movement of live birds or fomites. The application of a range of spatial statistical and geostatistical methods to analyse data from large HPAI outbreaks can provide insights into the contribution of these various introduction and spread pathways. As an example, results of analysis in the present study suggest that HPAI subtype 


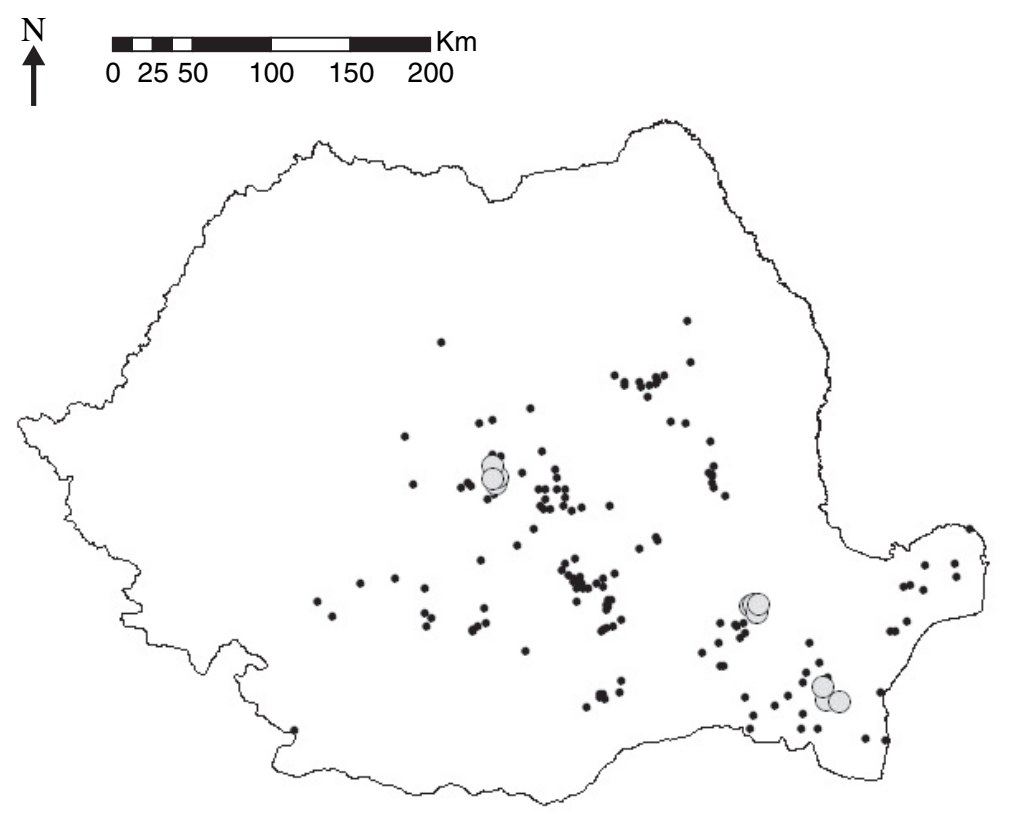

Figure 6. Clusters ( $)$ of highly-pathogenic avian influenza subtype H5N1 village outbreaks $(\bullet)$ in Romania, October 2005-June 2006, identified by the space-time permutation scan statistic.

H5N1 might have been introduced to eastern and southern Romania via migratory waterfowl during the autumn and winter of 2005, but that spread to central Romania in the spring and summer of 2006 might have occurred via the transportation of domestic poultry species. Although information on transmission route was not available in this study, a comparison of disease spread based on Euclidean distance versus the road network could be informative. We believe that all outbreaks of HPAI subtype $\mathrm{H} 5 \mathrm{~N} 1$ that occurred in Romania during the study period were reported. This conclusion is based on the dramatic clinical appearance of the disease in naive populations. The primary intervention methods deployed during this epidemic, following detection of each outbreak, were quarantine, depopulation, disinfection, and serological surveillance of sentinel chicken flocks. It is impossible to gauge the effectiveness of these interventions, since the outcome in the absence of such intervention is unknown. We focused on the day of first reporting of each outbreak, rather than for example the length of each outbreak. Study results have relevance to the design of preventive and surveillance programs for HPAI subtype H5N1, rather than specifically how such an epidemic might be most effectively controlled.

The role that wild, migratory birds played in the spread of HPAI subtype H5N1 from east Asia to central Asia, the Middle East, Europe, and Africa during 2005 to 2006 is still debated. Circumstantial evidence has implicated migratory birds, in some cases, as the mechanism of spread. For example, subtype H5N1 virus was detected at Erkhel Lake in Mongolia during the summer of 2005; because of the remoteness of this location and absence of domestic poultry in the area, introduction of this subtype via human activities was ruled-out. The best explanation was introduction via migratory wild birds [20]. In the northern hemisphere, influenza virus infection rates are highest in waterfowl in late summer and autumn, during southern migrations from breeding areas [13]. The 
first Romanian outbreaks of HPAI subtype H5N1 were reported on 7 October 2005 from two villages (60 km apart) located within the Danube River Delta region of southeast Romania. Coincidently, the first outbreak of subtype H5N1 in nearby Turkey was reported on 5 October, from the north-west region of the country [5]. Based on genetic analysis of the neuramidase (NA) gene of isolates from this first Turkish outbreak, the virus responsible was very similar to the A7 Great Black Headed Gull/Qinghai/1/05 lineage, and thus a link with central Asian outbreaks during 2005 was established. Considering the autumn migratory flyways of some Anatidae (ducks, swans and geese) from the West Siberian Lowlands and results of the analysis of outbreak data, it is likely that wild birds played a role in the introduction of subtype H5N1 to Romania during October-December $2005^{2}$.

Southeastern Romania is unique: it is bordered to the east by the Black Sea and is characterized by the Danube River Delta. There is an extensive wetland system, forming a major breeding area and point of congregation for migratory birds on the Black Sea-Mediterranean flyway (which extends from west Africa to central Asia). In this region, poultry production is almost entirely based at the village level. It is common for birds to have free range during the daylight hours, and contact with wild birds is likely at sites such as ponds and lakes. The movement of live poultry between villages is uncommon, and live bird markets do not exist.

At least 23 species of Anatidae overwinter in the Black Sea region ${ }^{3}$. Romania lies entirely within the European Siberia/Black

\footnotetext{
${ }^{2}$ Food and Agriculture Organisation, EMPRES Watch: Evolution of highly pathogenic avian influenza type H5N1 in Europe: review of disease ecology, trends and prospects of spread in autumnwinter 2006, http://www.fao.org/docs/eims/upload/ 214878/W_europe_oct06_hpai.pdf [consulted 25 June 2007].

${ }^{3}$ Waterbird Population Estimates, 2006, www.wetlands.org [consulted 16 June 2007].
}

Sea-Mediterranean flyway ${ }^{4}$, but the main routes of wild bird migration in Romania are not precisely known. More importantly, the migratory routes of wild birds vary by species. HPAI subtype H5N1 virus was reportedly isolated in Romania during 2005-2006 from four Anatidae species with a breeding range that extends to West Siberia: Cygnus cygnus (Whooper Swan), Anas penelope (Eurasian Wigeon), Anas crecca (Common Teal), and Anas acuta (Northern Pintail). A high mortality rate was observed in swans in the Danube River Delta during the epidemic. C. cygnus is a Palearctic species that is almost entirely migratory. It consists of probably four subpopulations, one of which breeds in western Siberia and winters in the Black Sea and east Mediterranean. The Danube River Delta is an important wintering site: 20$30 \%$ of the population can be found here during winter [23]. C. cygnus depart from their breeding grounds in mid- to late-September. Of interest to note is that the first detection of HPAI subtype H5N1 in the British Isles was isolation from a dead Whooper Swan sampled in March 2006, in Fife, Scotland [2]. Another species of interest with respect to HPAI subtype H5N1 virus spread is the Mute Swan (Cygnus olor). The first incursion of HPAI subtype H5N1 virus in the Czech Republic was detected in this species [19]. Although the distribution of C. olor includes Romania, most of this species breeding in the Black Sea region undertake only limited migratory movements, leaving inland breeding

\footnotetext{
${ }^{4}$ Isakov Y.A., MAR project and conservation of waterfowl breeding in the USSR, in: Salverda Z. (Ed.), Proc. Second European meeting on wildfowl conservation, Noordwijk aan Zee, The Netherlands, 9-14 May 1966, pp. 125-138. Ministry of Cultural Affairs, Recreation and Social Welfare, The Netherlands, 1967.

${ }^{5}$ Ontanu G., Nicolae S., Savuta G., Stochici A., Hulea M., Matei F.G., Risk analysis for avian influenza - risk identification and emission assessment, in: Proceedings of the 11th Symposium of the ISVEE, Cairns, Australia, 2006, http://www.sciquest.org.nz/crusher_download.asp? article $=10002812$ [consulted 16 June 2007].
} 
areas to winter on the coasts of the Black Sea [23].

If the H5N1 subtype was introduced to Romania via migratory waterfowl, winter conditions might have initially limited its spread to southeastern Romania. Thus, only localized outbreaks occurred as the result of contact between wild birds and domestic village poultry at common feeding sites, such as ponds and lakes. In this study, interpolation facilitated identification of the central role of the environment and landscape (specifically the Danube River Delta) in the introduction and initial spread of HPAI subtype H5N1 in Romania during the 2005-2006 epidemic.

The overall direction of spread of HPAI subtype H5N1 outbreaks was westerly, but the direction of spread within both the early and later phases of the epidemic was southerly. Within Romania, wild bird migration in general follows the Danube River (Fig. 4), which would explain the southerly direction of spread in the first two phases of the epidemic. The spatio-temporal distributions of reported cases of the avian influenza H5N1 in southern China during a six-week period (19 January through 9 March 2004) has been investigated [21]. A major northeastsouthwest distribution of the avian influenza H5N1 cases was observed. This directional finding is consistent with the major migratory bird routes in east Asia. The reason for the southerly direction of spread in the last phase of the epidemic in Romania is unknown. The movement of domestic poultry within central Romania could be an explanation.

Although migrating birds are a logical link between seemingly isolated outbreaks of HPAI subtype H5N1 in domestic poultry populations, isolation of the $\mathrm{H} 5 \mathrm{~N} 1$ subtype from healthy wild birds is rare; and this observation seemingly argues against wild migratory birds playing a major role in the spread of the infection (aptly summarized in the refrain, "Dead ducks don't fly"). In addition, the H5N1 subtype has not spread via known migratory flyways, as expected, to locations such as the Philippines and Taiwan, and Alaska. Whilst outbreaks of influenza in domestic poultry continue to be blamed on contact with free-range and wild birds, based on the circumstantial evidence of co-location of wild birds and domestic poultry in time and space [7], illegal movements of poultry and other avian species have been documented as contributing to $\mathrm{H} 5 \mathrm{~N} 1$ spread in several instances [20,24]. Live bird markets have also been the presumed source of avian influenza outbreaks in domestic poultry, notably in the United States $[6,10,16,18,22]$ and in Asia [24].

The route of wild bird migration within Romania (following in general the Danube River; Fig. 4) is not consistent with the spread of HPAI subtype H5N1 from southeastern to central Romania. An explanation might be the movement of domestic poultry (for sale or consumption) between the regions. The use of spatial statistical and geostatistical methods in this study highlights a potentially important transition zone in eastern Romania, where a critical mass of infected poultry might have developed and promoted the large number of outbreaks reported during the spring and summer of 2006 from central Romania. Using only mean center and directional deviational ellipse statistics, the distributions of phases 1 and 2 were clearly differentiated from phase 3. Interpolation of outbreak day using kriging produced an isopleth map that is also consistent with a phased epidemic. Kriging is a visualisation technique that can assist in the identification of disease spread patterns. Although it is a data-driven technique, the lag size and number of lags must be selected somewhat subjectively to produce a semivariogram that can be parametrically modeled. In this study a lag of $25 \mathrm{~km}$ was selected as the distance which, in combination with a lag number of 15 , produced a semivariogram that could successfully be modelled. A range of lag sizes were considered, but the resulting semivariograms were not as amenable to modelling as the semivariogram produced using a lag size of $25 \mathrm{~km}$. The mean distance between outbreak locations in this study was $9.5 \mathrm{~km}$, so the choice of a lag distance less than approximately $10-15 \mathrm{~km}$ would have provided less information for semivariogram modelling.

Page 10 of 12 (page number not for citation purpose) 
A rule-of-thumb for determining the lag size is that the product of lag size and number of lags should be approximately half the maximum distance of all possible pairs of locations [3,4]. In this study, the maximum distance between pairs of outbreak locations was $534 \mathrm{~km}$. Using the rule-of-thumb and a fixed number of 15 lags, an appropriate lag size might be approximately $18 \mathrm{~km}$. Modelling of the semivariogram allows a better understanding of the spatial nature of data, and allows interpolation to be performed. The reason for using an interpolation technique, such as kriging, is to reveal disease occurrence patterns that might not be visualized by simply dot or choropleth maps. This process assists in generating hypotheses regarding disease spread (for example, wild migratory birds versus transport of infected poultry). However, the resulting isopleth maps should not be interpreted as a prediction of disease outbreak occurrence at a given location, or as a quantitative estimation of outbreak day.

A notable cluster of four village outbreaks in southeast Romania, 18 November and 1 December 2005, was detected using the spatial scan statistic. A cluster of nine outbreaks, also in southeast Romania, between 20 November 2005 and 7 January 2006 was detected using the local indicator of spatial autocorrelation. Cluster detection statistics are not powerful, and it has been recommended that more than one technique be used to analyse the distribution of disease events $[29,30]$. The results of the analytical approaches used in this study were, in general, consistent and complementary. Considering the location and timing of these clusters (Figs. 5 and 6), descriptive spatial statistics (Fig. 2), and visualization of the epidemic evolution (Fig. 4), one of more of these outbreaks might have been the origin for the outbreaks that occurred in central Romania during 2006, a period during which the HPAI subtype H5N1 epidemic apparently changed from predominantly being associated with migratory waterfowl to a more complex epidemic in which the movement of poultry and poultry products might have been a factor. The study of landscape, animal production, and human factors in such epidemic transition zones can help us to understand why large outbreaks of HPAI subtype $\mathrm{H} 5 \mathrm{~N} 1$ occur in countries previously free of infection. Spatial statistics and geostatistical methods have a role to play in helping us resolve the debate over the role of migratory wild birds versus the movement of poultry and poultry products in the global spread of HPAI subtype H5N1.

Acknowledgements. Support for the acquisition and analysis of the data used in this study was provided by the United States Department of Agriculture Foreign Agriculture Service.

\section{REFERENCES}

[1] Alexander D.J., A review of avian influenza in different bird species, Vet. Microbiol. (2000) 74:3-13.

[2] Blissitt M., H5N1 avian influenza virus found in a Whooper swan in Scotland, Government Veterinary Journal (2007) 17:10-13.

[3] Carrat F., Valleron A.J., Epidemiologic mapping using the "kriging" method: application to an influenza-like illness epidemic in France, Am. J. Epidemiol. (1992) 135:1293-1300.

[4] Clark I., Practical geostatistics, Applied Science Publishers Ltd, London, UK, 1982.

[5] Coven F., The situation of highly pathogenic avian influenza (HPAI) outbreaks in Turkey, J. Vet. Med. B Infect. Dis. Vet. Public Health (2006) 53:34.

[6] Davison S., Eckroade R.J., Ziegler A.F., A review of the 1996-98 nonpathogenic H7N2 avian influenza outbreak in Pennsylvania, Avian Dis. (2003) 47:823827.

[7] De Benedictis P., Joannis T.M., Lombin L.H., Shittu I., Beato M.S., Rebonato V., Cattoli G., Capua I., Field and laboratory findings of the first incursion of the Asian H5N1 highly pathogenic avian influenza virus in Africa, Avian Pathol. (2007) 36:115-117.

[8] Delogu M., De Marco M.A., Donatelli I., Campitelli L., Catelli E., Ecological aspects of influenza A virus circulation in wild birds of the western palearctic, Vet. Res. Comm. (2003) 27:101106.

[9] Dinh P.N., Long H.T., Tien N.T.K., Hien N.T., Mai L.T.Q., Phong L.H., Tuan L.V., Tan H.V., Nguyen N.B., Tu P.V., Phuong N.T.M., Risk factors for human infection with avian influenza A H5N1, Vietnam, 2004, Emerging Infect. Dis. (2006) 12:1841-1847.

[10] Dunn P.A., Wallner-Pendleton E.A., Lu H., Shaw D.P., Kradel D., Henzler D.J., Miller P., Key D.W., 
Ruano M., Davison S., Summary of the 2001-02 Pennsylvania H7N2 low pathogenicity avian influenza outbreak in meat type chickens, Avian Dis. (2003) 47:812-816.

[11] Jacquez G.M., Oden N., User manual for Stat!: Statistical software for the clustering of health events, BioMedware, Ann Arbor, MI, USA, 1994.

[12] Khawaja J.Z., Naeem K., Ahmed Z., Ahmad S., Surveillance of avian influenza viruses in wild birds in areas adjacent to the epicenter of an outbreak in Federal Capital Territory of Pakistan, Int. J. Poultry Sci. (2005) 4:39-43.

[13] Krauss S., Walker D., Pryor S.P., Niles L., Chenghong L., Hinshaw V.S., Webser R.G., Influenza A viruses of migrating wild aquatic birds in North America, Vector Borne Zoonotic Dis. (2004) 4:177189.

[14] Kulldorff M., Heffernan R., Hartman J., Assunção R.M., Mostashari F., A space-time permutation scan statistic for the early detection of disease outbreaks, PLoS Medicine (2005) 2:216-224.

[15] Liu J., Xiao H., Lei F., Zhu Q., Qin K., Zhang X.W., Zhang X.L., Zhao D., Wang G., Feng Y., Ma J., Liu W., Wang J., Gao G.F., Highly pathogenic H5N1 influenza virus infection in migratory birds, Science (2005) 309:1206.

[16] Lu H., Dunn P.A., Wallner-Pendleton E.A., Henzler D.J., Kradel D.C., Liu J., Shaw D.P., Miller P., Investigation of H7N2 avian influenza outbreaks in two broiler breeder flocks in Pennsylvania, 2001-02, Avian Dis. (2004) 48:26-33.

[17] Morgan I.R., Kelly A.P., Epidemiology of an avian influenza outbreak in Victoria in 1985, Aust. Vet. J. (1990) 67:125-128.

[18] Mullaney R., Live-bird market closure activities in the northeastern United States, Avian Dis. (2003) 47:1096-1098.

[19] Nagy A., Machova J., Hornickova J., Tomci M., Nagl I., Horyna B., Holko I., Highly pathogenic avian influenza virus subtype H5N1 in Mute swans in the Czech Republic, Vet. Microbiol. (2007) 120: 9-16.

[20] Normile D., Avian influenza. Are wild birds to blame? Science (2005) 310:426-428.

[21] Oyana T.J., Dai D., Scott K.E., Spatiotemporal distributions of reported cases of the avian influenza H5N1 (bird flu) in southern China in early 2004, Avian Dis. (2006) 50:508-515.
[22] Pelzel A.M., McCluskey B.J., Scott A.E., Review of the highly pathogenic avian influenza outbreak in Texas, 2004, J. Am. Vet. Med. Assoc. (2006) 228:1869-1875.

[23] Scott D.A., Rose P.M., Atlas of Anatidae populations in Africa and western Eurasia, Wetlands International Publication No. 41, Wageningen, the Netherlands, 1996.

[24] Sims L.D., Domenech J., Benigno C., Kahn S., Kamata A., Lubroth J., Martin V., Roeder P., Origin and evolution of highly pathogenic H5N1 avian influenza in Asia, Vet. Rec. (2005) 157:159164.

[25] Stallnecht D.E., Shane S.M., Host range of avian influenza virus in free-living birds, Vet. Res. Comm. (1988) 12:125-141.

[26] Sturm-Ramirez K.M., Ellis T.M., Bousfield B., Bissett L., Dyrting K., Rehg J.E., Poon L., Guan Y., Peiris J.S.M., Webster R.G., Reemerging H5N1 influenza viruses in Hong Kong in 2002 are highly pathogenic to ducks, J. Virol. (2004) 78:48924901.

[27] Velkers F.C., Bouma A., Matthijs M.G.R., Koch G., Westendorp S.T., Stegeman J.A., Outbreak of avian influenza H7N3 on a turkey farm in the Netherlands, Vet. Rec. (2006) 159:403-405.

[28] Ward M.P., GIS-based avian influenza surveillance systems for village poultry in Romania, Vet. Ital. (2007) 43:483-490.

[29] Ward M.P., Carpenter T.E., Analysis of timespace clustering in veterinary epidemiology, Prev. Vet. Med. (2000) 43:225-237.

[30] Ward M.P., Carpenter T.E., Techniques for analysis of disease clustering in space and in time in veterinary epidemiology, Prev. Vet. Med. (2000) 45:257-284

[31] Webster R.G., Hulse D.J., Microbial adaptation and change: avian influenza, Rev. -Off. Int. Epizoot. (2004) 23:453-465.

[32] Webster R.G., Bean W.J., Gorman O.T., Chambers T.M., Kawaoka Y., Evolution and ecology of influenza A viruses, Microbiol. Rev. (1992) 56:152179.

[33] Woebser G.A., Avian influenza, Newcastle disease, and other paramyxoviruses, in: Diseases of wild waterfowl, Plenum press, New-York, USA, 1997, pp. $29-41$. 